\title{
Trombose aguda de aneurisma de artéria femoral isolado: relato de caso
}

\author{
Acute thrombosis of isolated femoral artery aneurysm: case report
}

\author{
André Hideo Motoki ${ }^{1}$, André Ricardo Girardi ${ }^{1}$, Bruno Rick Ogata ${ }^{1}$, Dionizio Baroni Júnior ${ }^{1}$, \\ Fernando Yoshio Lara Shimizu ${ }^{1}$, Vitor Teixeira Liutti ${ }^{1}$, Antônio Lacerda Santos Filho ${ }^{2}$, \\ Nelson Mesquita Júnior ${ }^{3}$
}

\section{Resumo}

Aneurismas de artéria femoral são raros, porém são o segundo tipo mais freqüente de aneurismas periféricos, sendo o aneurisma de artéria poplítea o mais comum. Normalmente, são de etiologia aterosclerótica. As complicações deste aneurisma são representadas por embolia, trombose e, mais raramente, a rotura. O objetivo deste trabalho é relatar um caso de um paciente com 59 anos, masculino, com queixa de dor súbita em membro inferior esquerdo associado à frialdade, palidez e ausência de pulsos. O ecocolordoppler evidenciou a presença de aneurisma trombosado da artéria femoral comum. O paciente foi submetido, com sucesso, à ressecção do aneurisma, com reconstrução do leito arterial.

Palavras-chave: Aneurisma, artéria femoral, trombose.

\section{Introdução}

A primeira descrição de um procedimento cirúrgico para tratamento de um aneurisma verdadeiro da artéria femoral foi realizada por Sir Astley Cooper, que, em 1808, fez a ligadura da artéria ilíaca externa para a correção do aneurisma da artéria femoral. Na tentativa de manter o fluxo arterial, Goyanes realizou em Madrid, em 1906, a primeira reconstrução arterial utilizando um enxerto venoso, o que foi repetido por Horgarth Pringle, na cidade de Glasgow, Escócia, em 1913 . Os aneurismas de artéria femoral são relativamente raros, sendo dez vezes menos freqüentes que os aneurismas de aorta. São mais freqüentes em pacientes do sexo masculino de

\begin{abstract}
Common femoral artery aneurysms are rare; however, they are the second most frequent type of peripheral aneurysm, popliteal artery aneurysms being the most common. They usually have atherosclerotic ethiology. The complications of this aneurysm are thromboembolism and, more rarely, rupture. This article aimed at reporting the case of a 59-year-old male patient with complaint of sudden pain in his left leg associated with coldness, paleness and absence of pulses. Color Doppler ultrasound showed a thrombosed aneurysm of the common femoral artery. The patient was successfully submitted to aneurysm resection with reconstruction of the arterial bed.
\end{abstract}

Keywords: Aneurysm, femoral artery, thrombosis.

idade avançada e tabagistas. Dentre os aneurismas periféricos, os aneurismas de artéria femoral são o segundo mais freqüente, após os aneurismas da artéria poplítea, com $(80 \% \text { dos aneurismas periféricos) })^{2,3}$.

As complicações isquêmicas dos aneurismas femorais são as mais comuns. A trombose aguda ocorre em cerca de 15 a $32 \%$ dos casos e ocasiona isquemia aguda, geralmente grave ${ }^{4}$. No entanto, se não houver progressão importante da trombose, pode haver compensação por circulação colateral. Nesses casos, há uma queixa comum de claudicação intermitente ${ }^{4}$.

Este trabalho tem por finalidade descrever um caso de trombose aguda de aneurisma de artéria femoral isolado, submetido a tratamento cirúrgico.

1. Acadêmico de Medicina (4ํano), Faculdade Evangélica do Paraná (FEPAR), Curitiba, PR.

2. Mestre, Clínica Cirúrgica, Universidade Federal do Paraná (UFPR), Curitiba, PR. Professor assistente, Disciplina de Angiologia e Cirurgia Vascular, FEPAR, Curitiba, PR.

3. Mestre, Clínica Cirúrgica, UFPR, Curitiba, PR. Professor adjunto, Disciplina de Anatomia, Angiologia e Cirurgia Vascular, FEPAR, Curitiba, PR.

Trabalho realizado na Disciplina de Angiologia e Cirurgia Vascular, Faculdade Evangélica do Paraná (FEPAR), Curitiba, PR.

Não foram declarados conflitos de interesse associados à publicação deste artigo.

Artigo submetido em 19.11.07, aceito em 19.10.08.

J Vasc Bras. 2008;7(4):376-379.

Copyright@ 2008 by Sociedade Brasileira de Angiologia e de Cirurgia Vascular 


\section{Relato de caso}

J.J.M., 59 anos, masculino, admitido no pronto socorro do Hospital Universitário Evangélico de Curitiba, foi encaminhado com queixa de dor de início súbito em membro inferior esquerdo, associado à frialdade, com 5 dias de evolução. Paciente negava história prévia de claudicação intermitente, angina ou infarto agudo do miocárdio. Não havia outras comorbidades associadas, como hipertensão arterial sistêmica ou diabetes. Também negava tabagismo ou etilismo.

Ao exame físico, o paciente apresentava o membro inferior esquerdo pálido e com ausência de pulsos, sem alterações de motricidade, mas com parestesia de pododáctilos e com pequeno aumento de volume nãopulsátil na região femoral esquerda.

Foi realizado ecocolordoppler, que mostrou dilatação aneurismática com presença de um trombo agudo ocluindo a luz da artéria femoral comum. Foi identificada reabitação na sua bifurcação, com fluxo monofásico na árvore arterial distal. $\mathrm{O}$ ecodoppler das artérias do membro inferior direito e de território aorto-ilíaco mostrou ausência de aneurismas e de placas ateromatosas em toda a sua extensão. O ecocardiograma transesofágico descartou a presença de trombos intracavitários.

O paciente foi submetido a tratamento cirúrgico, no qual foi confirmada a presença de um aneurisma de $3 \mathrm{x}$ $3 \mathrm{~cm}$ na artéria femoral comum, sem envolvimento da bifurcação. As porções proximal e distal ao aneurisma apresentavam artérias íntegras. Foi então realizada a ressecção do aneurisma, trombectomia distal (Figuras 1 e 2), com uso de cateter de Fogarty (que progrediu pela árvore arterial distal sem dificuldades) e confecção de uma ponte fêmoro-femoral, com prótese de Dacron $\mathrm{n}^{\circ}$ 10 com anastomoses terminoterminais (Figura 3), mantendo o fluxo para as artérias femoral superficial e profunda. O estudo anatomopatológico mostrou etiologia aterosclerótica. O paciente teve boa evolução, recebendo alta no quinto dia pós-operatório. Encontra-se assintomático, sem claudicação intermitente, com ponte pérvia e pulsos distais presentes. O tempo de seguimento é de 8 meses, sendo assintomático e com pulsos presentes.

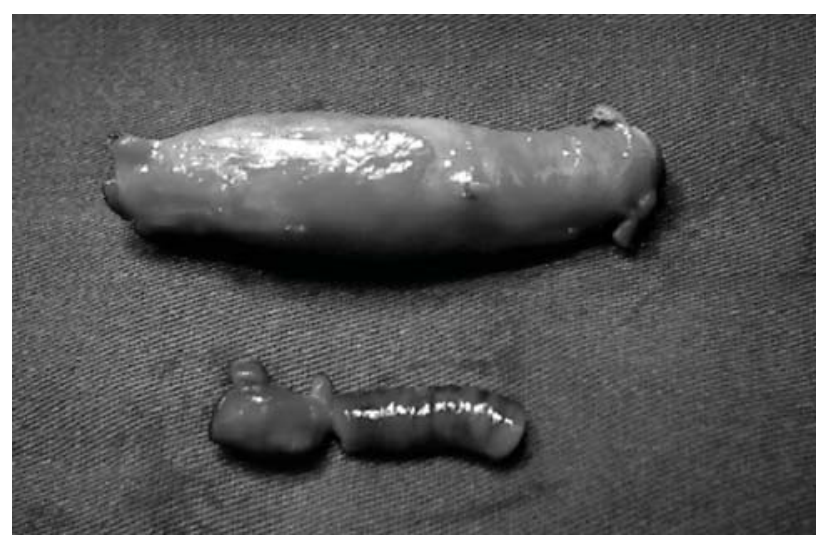

Figura 1 - Aneurisma ressecado

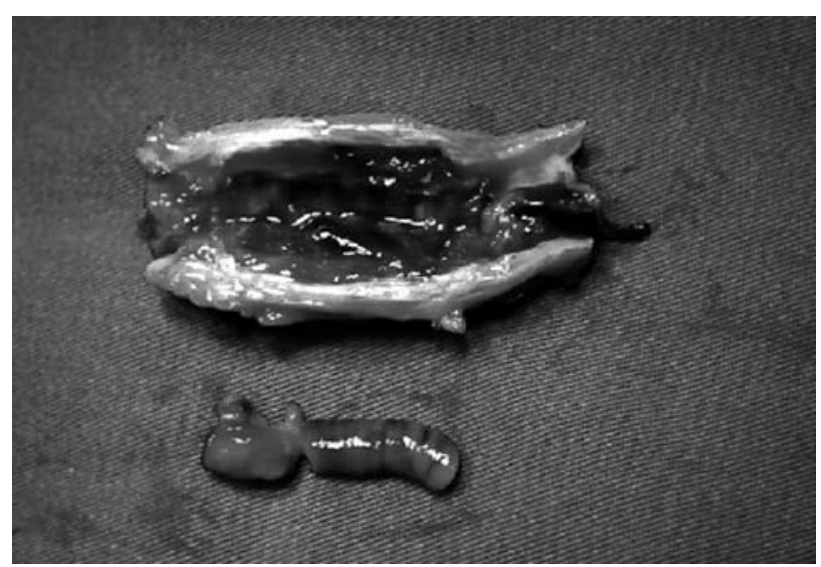

Figura 2 - Peça aneurismática aberta com detalhes do trombo

\section{Discussão}

Aneurismas periféricos são entidades raras e, quando presentes em membro inferiores, a artéria poplítea e a artéria femoral comum representam o primeiro e o segundo territórios arteriais mais acometidos. Com a progressão da doença, podem surgir complicações, tais como embolia, trombose e rotura do aneurisma ${ }^{5,6}$. De acordo com a literatura, a trombose em aneurismas de artéria femoral comum ocorre em $32 \%$ dos casos ${ }^{2}$.

A artéria mais acometida por aneurismas, em território femoral, é a femoral comum. As artérias femoral superficial e femoral profunda são raramente acometidas por essa patologia ${ }^{2,6-9}$. Na maioria das vezes, a origem de tal patologia é aterosclerótica, decorrente de alterações degenerativas da parede do vaso influenciadas principalmente pelo tabagismo ${ }^{1,2,5,6}$. O aneurisma é 


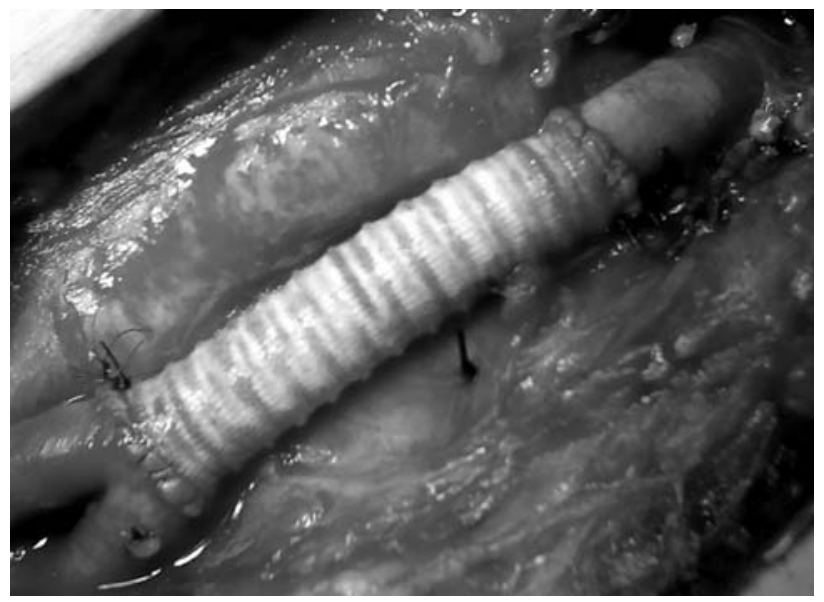

Figura 3 - Interposição fêmoro-femoral de uma prótese de Dacron $\mathrm{n}^{\circ} 10$, com anastomose terminoterminal

denominado aterosclerótico quando o enfraquecimento da parede é secundário ao processo de aterosclerose. Esse enfraquecimento também pode ocorrer secundário a infecções crônicas (como os aneurismas micóticos e sifilíticos) ou por alteração do tecido conjuntivo (doença de Behcet, LES, arterites) ${ }^{8-10}$. A denominação de aneurisma aterosclerótico também se estende a pacientes com dilatações que não estão associadas à doença aterosclerótica moderada ou avançada em outros vasos e que também não apresentam outras causas identificáveis para justificar o enfraquecimento da parede arterial ${ }^{7,11}$. Os aneurismas ateroscleróticos da femoral constituem pouco mais de $1 / 3$ de todos os aneurismas periféricos ${ }^{1}$. No entanto, aneurismas em território femoral podem apresentar etiologia iatrogênica, resultantes de rotura de sutura ou desgarramento de uma prótese anastomosada na artéria femoral, ou que, ainda, podem ser conseqüência de cateterismos. Outros fatores incluem a vibração da parede e fadiga arterial, condições essas relacionadas ao estresse de movimentação crônica durante uma flexão forçada da coxa e turbulência do fluxo sanguíneo. Doenças do colágeno, infecções crônicas micóticas e sifilíticas também podem estar associadas a aneurisma de artéria femoral ${ }^{4,8,9}$.

Os aneurismas de artéria femoral podem aparecer em sincronia com outros aneurismas em 50\% dos casos, Sua associação com aneurisma de aorta é encontrada em 33 a $63,8 \%$ dos casos, sendo que 18 a $72 \%$ podem ser bilaterais $^{1,3,4}$. No caso relatado, não foram encontrados outros aneurismas.

Essa patologia é assintomática em até metade dos casos, mas pode existir dor local ou sintomas decorrentes de isquemia de membro inferior ${ }^{1,3}$. A compressão de estruturas nervosas gera dor em região femoral ou face anterior da coxa, ou ainda uma irradiação distal pode cursar com paresia de grupos musculares. A veia femoral pode ser comprimida, gerando edema e/ou outros sinais e sintomas de estase venosa ${ }^{12}$. O diagnóstico desse tipo de aneurisma pode ser feito clinicamente por palpação de abaulamento pulsátil na região femoral e expansível em todos os sentidos, caracterizando um aneurisma arterial ${ }^{1,2}$.

Segundo Arroyo-Bielsa et al., de 68 aneurismas de artéria femoral encontrados em um período de 14 anos, evidenciou-se $10,2 \%$ de trombose crônica e $4,4 \%$ de trombose aguda ${ }^{1}$.

Classificando os aneurismas desse território, pode-se descrever dois tipos: 1) acometendo somente a artéria femoral comum, sem comprometer a bifurcação; e 2) englobando a bifurcação ${ }^{3,5,12}$.

Apesar de o diagnóstico desses aneurismas ser eminentemente clínico, o uso de métodos não-invasivos, como o ecocolordoppler, contribuem para a confirmação do diagnóstico $2,4,12,13$. Outros exames, tais como a tomografia axial computadorizada, ressonância magnética e arteriografia, também são úteis ${ }^{2,5}$.

A cirurgia será indicada para todos os aneurismas sintomáticos com ou sem complicações, assim como para os assintomáticos com mais de $2,5 \mathrm{~cm}$ de diâmetro $^{7,11}$.

No presente caso, foi realizada ressecção do aneurisma com passagem de cateter de Fogarty no leito distal, com posterior colocação de uma prótese de Dacron terminoterminal na artéria femoral. Em quadros agudos de isquemia em membros, o diagnóstico etiológico precoce é de grande importância, tanto para o prognóstico da doença quanto para a escolha do tratamento ${ }^{2}$.

Conclui-se que, além de ser um tipo de aneurisma incomum, a trombose é rara, pois sua localização tende a ser diagnosticada pelo próprio paciente ou por um médico em exame de rotina antes dessa complicação. A 
boa evolução do caso também foge do padrão esperado, pelo tempo prolongado de isquemia e ausência de doença aterosclerótica significativa.

\section{Referências}

1. Arroyo-Bielsa A, Rodríguez-Montalbán AI, Sáinz-González F, et al. Aneurismas ateroescleróticos de la arteria femoral común. Angiología. 1995;5:251-6.

2. Puech-Leão P, Kauffman P. Aneurismas arteriais. São Paulo: Byk; 1998.

3. Martínez-Ramos D, Villalba-Munera V, García-Calvo R, Miralles-Tena JM, Molina-Martínez J, Salvador-Sanchís JL. Trombosis venosa profunda como manifestación clínica inicial de un aneurisma de la arteria femoral común. Angiología. 2006;58:331-4.

4. Levi N, Schroeder TV. Arteriosclerotic femoral artery aneurysms: a short review. J Cardiovasc Surg (Torino). 1997;38:335-8.

5. Kolde E, Rocha MF, Franco FC, Ornato SJTA. Ruptura de aneurisma de arteria femoral. Cir Vasc Angiol. 1998;14:40-2.

6. Harbuzariu C, Duncan AA, Bower TC, Kalra M, Gloviczki P. Profunda femoris artery aneurysm: association with aneurysmal disease and limb ischemia. J Vasc Surg. 2008;47:31-5; discussion 34-5.
7. Galindo CC, Lima CA, Cardoso JE, Galindo Filho G, Costa VS, Penha FM. Aneurisma aterosclerótico isolado da artéria femoral superficial - relato de caso. J Vasc Bras. 2003;2:145-7.

8. Levi N, Schroeder TV. Rupture of true profunda femoris artery aneurysms. Two new cases. J Cardiovasc Surg (Torino). 1996;37:117-8.

9. Lima IA, Lima MF, Bernardes MV. Aneurisma isolado de artéria femoral profunda. Relato de caso e revisão bibliográfica. J Vasc Bras. 2003;2:333-8.

10. Koç Y, Güllü I, Akpek G, et al. Vascular involvement in Behçet's disease. J Rheumatol. 1992;19:402-10.

11. Rigdon EE, Monajjen N. Aneurysms of the superficial femoral artery: a report of two cases and review of the literature. J Vasc Surg. 1992;16:790-3.

12. Maffei FHA, Lastória S, Yoshida WB, Rollo HA. Doenças vasculares periféricas. 3ª ed. São Paulo: Medsi; 2002.

13. Corriere MA, Guzman RJ. True and false aneurysms of the femoral artery. Semin Vasc Surg. 2005;18:216-23.

Correspondência:

Nelson Mesquita Júnior

Rua Deputado Heitor Alencar Furtado, 1819/1302, Mossunguê

CEP 81200-110 - Curitiba, PR

Tel.: (41) 3336.6966, (41) 9994.5167

E-mail: nelsonmesquita@terra.com.br 